\title{
Electron Detachment and Subsequent Structral Changes of Water Clusters
}

\author{
Susanta Das ${ }^{a}$, Turbasu Sengupta ${ }^{a}$, Achintya Kumar Dutta ${ }^{a}$ and Sourav Pal ${ }^{b *}$ \\ aPhysical Chemistry Division, CSIR-National Chemical Laboratory, Pune 411008, India. \\ ${ }^{b}$ Department of Chemistry, Indian institute of Technology Bombay, Powai, Mumbai 400076 India
}

Submitted to J. Phys. Chem. A

Supplementary Data

\begin{tabular}{|rrrr|rrrr|}
\hline \multicolumn{3}{c}{ Neutral } & \multicolumn{3}{c|}{ Dimer } \\
\hline $\mathrm{O}$ & -1.487914 & -0.032912 & 0.058757 & $\mathrm{O}$ & 1.308637 & 0.054298 & -0.028174 \\
$\mathrm{H}$ & -1.857023 & 0.400395 & -0.714814 & $\mathrm{O}$ & -1.212379 & -0.019754 & 0.051425 \\
$\mathrm{H}$ & -0.529110 & 0.035261 & -0.060901 & $\mathrm{H}$ & 1.885366 & -0.680051 & 0.278056 \\
$\mathrm{O}$ & 1.416322 & 0.030814 & -0.055450 & $\mathrm{H}$ & -0.168886 & -0.066527 & -0.013636 \\
$\mathrm{H}$ & 1.760428 & -0.866408 & -0.007930 & $\mathrm{H}$ & -1.567305 & 0.873425 & -0.128535 \\
$\mathrm{H}$ & 1.761908 & 0.464052 & 0.731153 & $\mathrm{H}$ & -1.676858 & -0.675092 & -0.504896 \\
\hline
\end{tabular}

\begin{tabular}{|rrrr|rrrr|}
\hline \multicolumn{7}{|c}{ Neutral } & \multicolumn{1}{c|}{ Cation } \\
\hline $\mathrm{O}$ & -1.478742 & 0.655054 & 0.047145 & $\mathrm{O}$ & -1.976759 & 0.503348 & -0.007574 \\
$\mathrm{H}$ & -0.607451 & 1.091826 & -0.031997 & $\mathrm{O}$ & -0.008325 & -0.982279 & -0.039044 \\
$\mathrm{H}$ & -2.034437 & 1.073041 & -0.621694 & $\mathrm{O}$ & 2.116475 & 0.540366 & 0.018765 \\
$\mathrm{O}$ & 0.168796 & -1.607504 & -0.062516 & $\mathrm{H}$ & -2.399168 & 0.895564 & 0.770793 \\
$\mathrm{H}$ & 0.080704 & -2.223472 & 0.675335 & $\mathrm{H}$ & -2.659990 & 0.425798 & -0.690390 \\
$\mathrm{H}$ & -0.634972 & -1.050983 & -0.024707 & $\mathrm{H}$ & -0.025148 & -1.728675 & 0.583128 \\
$\mathrm{O}$ & 1.309112 & 0.949662 & 0.052891 & $\mathrm{H}$ & -0.870453 & -0.373126 & 0.035825 \\
$\mathrm{H}$ & 1.983747 & 1.178041 & -0.597958 & $\mathrm{H}$ & 3.012453 & 0.273924 & -0.275528 \\
$\mathrm{H}$ & 1.225648 & -0.024203 & 0.005551 & $\mathrm{H}$ & 0.857040 & -0.468501 & 0.018222 \\
\hline
\end{tabular}

\begin{tabular}{|rrrr|rrrr|}
\hline \multicolumn{3}{c}{ Neutral } & \multicolumn{3}{c|}{ Tetramer } \\
\hline $\mathrm{O}$ & -1.338414 & 1.394733 & -0.005082 & $\mathrm{O}$ & 3.052616 & -0.762922 & -0.125850 \\
$\mathrm{O}$ & -1.394733 & -1.338415 & 0.005082 & $\mathrm{O}$ & -0.932341 & 0.394074 & -0.869813 \\
$\mathrm{O}$ & 1.394733 & 1.338405 & 0.005081 & $\mathrm{O}$ & 0.977396 & 0.848537 & 0.585102 \\
$\mathrm{O}$ & 1.338415 & -1.394723 & -0.005081 & $\mathrm{O}$ & -2.910764 & -0.575206 & 0.381086 \\
$\mathrm{H}$ & -1.842338 & 1.735366 & 0.742439 & $\mathrm{H}$ & 1.815844 & 0.374703 & 0.387102 \\
$\mathrm{H}$ & -1.495652 & 0.420411 & -0.014141 & $\mathrm{H}$ & 3.955450 & -0.833489 & 0.242827 \\
$\mathrm{H}$ & -0.420409 & -1.495643 & 0.014138 & $\mathrm{H}$ & -1.166393 & 1.082952 & -1.510707 \\
$\mathrm{H}$ & -1.735372 & -1.842339 & -0.742435 & $\mathrm{H}$ & -1.761689 & 0.059381 & -0.373996 \\
$\mathrm{H}$ & 1.735368 & 1.842327 & -0.742439 & $\mathrm{H}$ & -0.104808 & 0.664471 & -0.238611 \\
$\mathrm{H}$ & 0.420411 & 1.495646 & 0.014140 & $\mathrm{H}$ & 1.187668 & 1.706859 & 0.976285 \\
$\mathrm{H}$ & 1.495639 & -0.420399 & -0.014138 & $\mathrm{H}$ & -3.281157 & -1.433209 & 0.129932 \\
$\mathrm{H}$ & 1.842340 & -1.735361 & 0.742436 & $\mathrm{H}$ & -3.611267 & -0.105759 & 0.854956 \\
\hline
\end{tabular}

\begin{tabular}{|c|c|c|c|c|c|c|c|}
\hline \multicolumn{8}{|c|}{ Pentamer } \\
\hline & & Neutral & & & & Cation & \\
\hline $\mathrm{O}$ & 0.963258 & -2.079592 & 0.225685 & 0 & -1.454537 & 1.277655 & 0.408860 \\
\hline
\end{tabular}




\begin{tabular}{|rrrr|rrrr|}
\hline $\mathrm{O}$ & -1.680761 & -1.580188 & -0.107818 & $\mathrm{O}$ & 0.910382 & 1.443910 & -0.379357 \\
$\mathrm{O}$ & -2.029142 & 1.108057 & 0.002114 & $\mathrm{O}$ & 2.831170 & -0.547207 & 0.045678 \\
$\mathrm{O}$ & 2.296176 & 0.263491 & -0.090813 & $\mathrm{O}$ & -2.516728 & -0.869126 & -0.288045 \\
$\mathrm{O}$ & 0.436544 & 2.244576 & 0.091213 & $\mathrm{O}$ & 0.115788 & -1.361227 & 0.228936 \\
$\mathrm{H}$ & 1.481853 & -1.245569 & 0.110196 & $\mathrm{H}$ & -1.901682 & 0.390785 & 0.091299 \\
$\mathrm{H}$ & 1.572375 & -1.870033 & -0.490943 & $\mathrm{H}$ & -1.548370 & 1.360957 & 1.371303 \\
$\mathrm{H}$ & 2.798513 & 0.373847 & -0.905487 & $\mathrm{H}$ & -3.263930 & -0.958312 & -0.895663 \\
$\mathrm{H}$ & 1.669730 & 1.026419 & -0.054266 & $\mathrm{H}$ & -1.888131 & -1.582238 & -0.477141 \\
$\mathrm{H}$ & 0.413335 & 3.032478 & -0.462321 & $\mathrm{H}$ & 1.082232 & -1.525805 & 0.265520 \\
$\mathrm{H}$ & -0.477126 & 1.867435 & 0.063047 & $\mathrm{H}$ & 3.445151 & -0.510602 & 0.795246 \\
$\mathrm{H}$ & -2.610646 & 1.261641 & 0.755160 & $\mathrm{H}$ & 3.400430 & -0.759988 & -0.710057 \\
$\mathrm{H}$ & -1.942147 & 0.125305 & -0.065663 & $\mathrm{H}$ & 1.664410 & 0.850643 & -0.182359 \\
$\mathrm{H}$ & -1.957598 & -2.073552 & -0.887617 & $\mathrm{H}$ & 1.270744 & 2.297861 & -0.654285 \\
$\mathrm{H}$ & -0.727296 & -1.805109 & 0.027357 & $\mathrm{H}$ & -0.452781 & 1.325379 & 0.141067 \\
\hline
\end{tabular}

\begin{tabular}{|rrrr|rrrr|}
\hline \multicolumn{7}{c}{ Neutral } & \multicolumn{3}{c|}{ Hexamer Book } \\
\hline $\mathrm{H}$ & 0.119966 & 1.479002 & 1.137954 & $\mathrm{O}$ & -0.737433 & 1.447258 & 0.976846 \\
$\mathrm{O}$ & -0.855879 & 1.585699 & 1.097663 & $\mathrm{O}$ & 1.479102 & 1.913660 & -0.397482 \\
$\mathrm{H}$ & -1.197703 & 0.813067 & 1.572713 & $\mathrm{O}$ & 2.702366 & -0.222420 & -0.392319 \\
$\mathrm{H}$ & 2.597483 & 1.571572 & 0.941542 & $\mathrm{O}$ & 1.127109 & -1.896042 & 0.549758 \\
$\mathrm{O}$ & 1.838850 & 0.983192 & 0.844982 & $\mathrm{O}$ & -1.514245 & -1.929116 & -0.262232 \\
$\mathrm{H}$ & 1.791115 & 0.756052 & -0.119036 & $\mathrm{O}$ & -3.037485 & 0.524446 & -0.468786 \\
$\mathrm{H}$ & -2.074703 & 1.218999 & -1.884748 & $\mathrm{H}$ & -0.934395 & 1.820112 & 1.846507 \\
$\mathrm{O}$ & -1.475478 & 0.677425 & -1.356355 & $\mathrm{H}$ & -1.598906 & 1.319967 & 0.539118 \\
$\mathrm{H}$ & -1.298211 & 1.193461 & -0.527405 & $\mathrm{H}$ & 1.378006 & -2.794536 & 0.802590 \\
$\mathrm{H}$ & 0.421569 & 0.234594 & -1.792241 & $\mathrm{H}$ & 2.060793 & -0.971124 & -0.036260 \\
$\mathrm{O}$ & 1.344735 & -0.022566 & -1.617531 & $\mathrm{H}$ & 1.927778 & 2.740896 & -0.174427 \\
$\mathrm{H}$ & 1.251426 & -0.880501 & -1.153766 & $\mathrm{H}$ & 0.644568 & 1.868002 & 0.140148 \\
$\mathrm{H}$ & 1.419173 & -1.312118 & 1.015371 & $\mathrm{H}$ & 2.208190 & 0.728442 & -0.387883 \\
$\mathrm{O}$ & 1.009317 & -1.958209 & 0.419804 & $\mathrm{H}$ & 3.000598 & -0.441617 & -1.289210 \\
$\mathrm{H}$ & 0.052695 & -1.850336 & 0.582610 & $\mathrm{H}$ & 0.190247 & -1.922207 & 0.250900 \\
$\mathrm{H}$ & -2.471083 & -1.969772 & 0.584505 & $\mathrm{H}$ & -2.044669 & -1.109050 & -0.390968 \\
$\mathrm{O}$ & -1.781146 & -1.294851 & 0.603255 & $\mathrm{H}$ & -3.907312 & 0.443356 & -0.048661 \\
$\mathrm{H}$ & -1.887729 & -0.788843 & -0.227655 & $\mathrm{H}$ & -3.233033 & 0.892220 & -1.343656 \\
\hline
\end{tabular}

\begin{tabular}{|rrrr|rrrr|}
\hline \multicolumn{7}{c}{ Neutral } & \multicolumn{3}{c|}{ Hexamer Cage } \\
\hline H & 1.464829 & 0.877532 & 0.831676 & $\mathrm{O}$ & 1.775581 & -1.542092 & -0.661576 \\
$\mathrm{O}$ & 0.968103 & 1.699352 & 0.567900 & $\mathrm{O}$ & -0.012390 & -1.309007 & 1.051775 \\
$\mathrm{H}$ & 1.543893 & 2.440393 & 0.791390 & $\mathrm{O}$ & 0.167760 & 1.223330 & 1.592582 \\
$\mathrm{H}$ & -2.250021 & 1.786908 & 1.263385 & $\mathrm{O}$ & 1.649918 & 1.161389 & -0.595458 \\
$\mathrm{O}$ & -1.762599 & 1.281177 & 0.601285 & $\mathrm{O}$ & -2.252752 & -1.173882 & -0.440659 \\
$\mathrm{H}$ & -0.821541 & 1.525506 & 0.737633 & $\mathrm{O}$ & -1.241481 & 1.684244 & -0.736551 \\
$\mathrm{H}$ & 1.614482 & -1.159052 & 0.319132 & $\mathrm{H}$ & 2.037650 & -0.607661 & -0.811588 \\
$\mathrm{O}$ & 2.148323 & -0.647725 & 0.985998 & $\mathrm{H}$ & 2.575887 & -2.084492 & -0.654212 \\
$\mathrm{H}$ & 2.073582 & -1.150672 & 1.806337 & $\mathrm{H}$ & -3.100057 & -1.323711 & 0.008084 \\
$\mathrm{H}$ & -2.705410 & -1.848155 & 0.340062 & $\mathrm{H}$ & -2.301452 & -1.742634 & -1.225364 \\
$\mathrm{O}$ & -1.843554 & -1.531405 & 0.637036 & $\mathrm{H}$ & 2.376152 & 1.801813 & -0.619758 \\
$\mathrm{H}$ & -1.923903 & -0.554688 & 0.657509 & $\mathrm{H}$ & 0.975238 & 1.521799 & -1.193771 \\
$\mathrm{H}$ & 0.449865 & 1.291989 & -1.264457 & $\mathrm{H}$ & -1.741500 & 2.446102 & -1.064131 \\
$\mathrm{O}$ & 0.036367 & 0.844933 & -2.026301 & $\mathrm{H}$ & -1.818714 & 0.919135 & -0.896446 \\
$\mathrm{H}$ & -0.903720 & 1.038928 & -1.905112 & $\mathrm{H}$ & 0.776823 & -1.509147 & 0.396673 \\
$\mathrm{H}$ & 0.389812 & -0.965553 & -1.454299 & $\mathrm{H}$ & -0.858184 & -1.385214 & 0.533903 \\
$\mathrm{O}$ & 0.540555 & -1.738173 & -0.873931 & $\mathrm{H}$ & 0.069696 & -0.338557 & 1.341827 \\
$\mathrm{H}$ & -0.315726 & -1.825548 & -0.409008 & $\mathrm{H}$ & -0.366509 & 1.604552 & 0.850133 \\
\hline
\end{tabular}




\begin{tabular}{|rrrr|rrrr|}
\hline \multicolumn{7}{c}{ Neutral } & \multicolumn{3}{c|}{ Hexamer Prism } \\
\hline H & 1.066110 & 1.454515 & 0.594853 & $\mathrm{O}$ & -1.862939 & -1.272127 & 0.364354 \\
$\mathrm{O}$ & 0.742860 & 1.980075 & -0.160811 & $\mathrm{O}$ & -1.841031 & 1.138928 & -0.062060 \\
$\mathrm{H}$ & 1.157471 & 1.521578 & -0.909513 & $\mathrm{O}$ & 0.502799 & 1.945821 & 0.428388 \\
$\mathrm{H}$ & 1.898425 & -0.480291 & 2.302277 & $\mathrm{O}$ & 1.629703 & -0.186163 & 1.485331 \\
$\mathrm{O}$ & 1.438290 & -0.257531 & 1.484250 & $\mathrm{O}$ & 0.619579 & -1.937586 & -0.463921 \\
$\mathrm{H}$ & 0.504479 & -0.594629 & 1.593139 & $\mathrm{O}$ & 0.930851 & 0.483454 & -1.719711 \\
$\mathrm{H}$ & -0.995650 & 1.632044 & -0.214837 & $\mathrm{H}$ & -0.993495 & -1.670234 & 0.109645 \\
$\mathrm{O}$ & -1.892016 & 1.219683 & -0.209413 & $\mathrm{H}$ & -2.157129 & -1.699698 & 1.180126 \\
$\mathrm{H}$ & -2.510282 & 1.945578 & -0.356711 & $\mathrm{H}$ & 0.837599 & -2.781988 & -0.882952 \\
$\mathrm{H}$ & 2.511899 & -1.059854 & -1.626506 & $\mathrm{H}$ & 0.808358 & -1.241796 & -1.129619 \\
$\mathrm{O}$ & 1.771350 & -0.567728 & -1.251054 & $\mathrm{H}$ & -1.915437 & 0.099710 & 0.198182 \\
$\mathrm{H}$ & 1.867414 & -0.645430 & -0.278386 & $\mathrm{H}$ & -2.559055 & 1.669287 & 0.318337 \\
$\mathrm{H}$ & -1.113398 & -1.424707 & 0.543741 & $\mathrm{H}$ & 2.382149 & -0.274431 & 2.085686 \\
$\mathrm{O}$ & -1.103804 & -1.049191 & 1.452325 & $\mathrm{H}$ & 1.548121 & -1.032900 & 1.011098 \\
$\mathrm{H}$ & -1.608059 & -0.229284 & 1.310447 & $\mathrm{H}$ & 1.832204 & 0.798169 & -1.890866 \\
$\mathrm{H}$ & -0.095459 & -1.151575 & -1.492211 & $\mathrm{H}$ & 0.451721 & 0.707189 & -2.532514 \\
$\mathrm{O}$ & -1.033849 & -1.356029 & -1.325591 & $\mathrm{H}$ & 1.008441 & 1.175290 & 0.831107 \\
$\mathrm{H}$ & -1.458222 & -0.480375 & -1.302931 & $\mathrm{H}$ & -0.909584 & 1.516447 & 0.187870 \\
\hline
\end{tabular}

\begin{tabular}{|rrrr|rrrr|}
\hline \multicolumn{7}{c}{ Neutral } & \multicolumn{3}{c|}{ Hexamer Ring } \\
\hline $\mathrm{O}$ & -2.616236 & 0.371230 & -0.342892 & $\mathrm{O}$ & -0.407153 & 1.877415 & 0.921565 \\
$\mathrm{O}$ & -0.922038 & 2.477258 & -0.190947 & $\mathrm{O}$ & -2.377496 & 1.012672 & -0.293697 \\
$\mathrm{O}$ & -1.656328 & -2.015134 & 0.509672 & $\mathrm{O}$ & -2.069261 & -1.428703 & -0.308861 \\
$\mathrm{O}$ & 0.921449 & -2.477279 & -0.191938 & $\mathrm{O}$ & 1.788743 & 1.833403 & -0.567914 \\
$\mathrm{O}$ & 2.616517 & -0.371642 & -0.341713 & $\mathrm{O}$ & 0.294721 & -2.462148 & 0.341091 \\
$\mathrm{O}$ & 1.656521 & 2.015549 & 0.508328 & $\mathrm{O}$ & 2.782121 & -0.882113 & -0.069235 \\
$\mathrm{H}$ & -3.017568 & 0.186694 & -1.199392 & $\mathrm{H}$ & -0.480423 & 2.586906 & 1.573092 \\
$\mathrm{H}$ & -2.263888 & -0.496488 & -0.025299 & $\mathrm{H}$ & 0.446995 & 1.987662 & 0.432899 \\
$\mathrm{H}$ & 1.445913 & -3.215992 & 0.133945 & $\mathrm{H}$ & 3.658410 & -1.304102 & -0.176188 \\
$\mathrm{H}$ & 1.540236 & -1.708243 & -0.251415 & $\mathrm{H}$ & 1.157004 & -2.021235 & 0.321122 \\
$\mathrm{H}$ & 3.019023 & -0.187512 & -1.197708 & $\mathrm{H}$ & 0.321920 & -3.075171 & 1.086994 \\
$\mathrm{H}$ & 2.263959 & 0.496293 & -0.025007 & $\mathrm{H}$ & -1.182873 & -1.815434 & -0.092200 \\
$\mathrm{H}$ & 1.742370 & 2.199718 & 1.449932 & $\mathrm{H}$ & -2.531510 & -2.041912 & -0.894018 \\
$\mathrm{H}$ & 0.714168 & 2.206407 & 0.281632 & $\mathrm{H}$ & -2.262343 & -0.041799 & -0.359301 \\
$\mathrm{H}$ & -1.446760 & 3.215338 & 0.135958 & $\mathrm{H}$ & -2.492261 & 1.402872 & -1.173386 \\
$\mathrm{H}$ & -1.540495 & 1.707996 & -0.251161 & $\mathrm{H}$ & -1.549090 & 1.445560 & 0.194473 \\
$\mathrm{H}$ & -0.714126 & -2.206121 & 0.282168 & $\mathrm{H}$ & 2.391056 & 1.089730 & -0.416708 \\
$\mathrm{H}$ & -1.741008 & -2.197791 & 1.451769 & $\mathrm{H}$ & 2.337832 & 2.572114 & -0.860989 \\
\hline
\end{tabular}

\begin{tabular}{|rrrr|rrrr|}
\hline \multicolumn{7}{c}{ Neutral } & \multicolumn{3}{c|}{ Cation } \\
\hline $\mathrm{O}$ & 2.835248 & 0.485046 & -0.349469 & $\mathrm{O}$ & -3.164983 & -0.039221 & -0.174852 \\
$\mathrm{H}$ & 3.742610 & 0.336586 & -0.057630 & $\mathrm{O}$ & -0.866809 & -0.476358 & -1.543287 \\
$\mathrm{H}$ & 2.309376 & 0.612955 & 0.476908 & $\mathrm{O}$ & 0.803167 & -1.981228 & -0.195928 \\
$\mathrm{O}$ & -0.260122 & -1.849294 & 1.195686 & $\mathrm{O}$ & 2.697995 & -0.273088 & -0.165801 \\
$\mathrm{H}$ & -0.429798 & -2.636303 & 1.727514 & $\mathrm{O}$ & 1.614457 & 1.889491 & -0.390455 \\
$\mathrm{H}$ & -1.149065 & -1.467084 & 0.989367 & $\mathrm{O}$ & -0.886826 & 1.554271 & 0.487003 \\
$\mathrm{O}$ & -1.062383 & 0.354435 & -1.875215 & $\mathrm{O}$ & -0.164599 & -0.515586 & 2.039047 \\
$\mathrm{H}$ & -1.224822 & 0.430362 & -2.823120 & $\mathrm{H}$ & -4.067604 & -0.334606 & -0.357126 \\
$\mathrm{H}$ & -0.271076 & -0.252439 & -1.779486 & $\mathrm{H}$ & -3.245155 & 0.897094 & 0.056408 \\
$\mathrm{O}$ & 0.953101 & -1.276348 & -1.357277 & $\mathrm{H}$ & 1.740733 & 2.395358 & -1.205456 \\
$\mathrm{H}$ & 1.733522 & -0.741823 & -1.091495 & $\mathrm{H}$ & 2.217526 & 0.716196 & -0.291514 \\
$\mathrm{H}$ & 0.659211 & -1.687955 & -0.523455 & $\mathrm{H}$ & 0.928260 & -2.894506 & -0.490385 \\
$\mathrm{O}$ & -2.486582 & -0.504888 & 0.409817 & $\mathrm{H}$ & 0.115353 & -1.571390 & -0.795103 \\
\hline
\end{tabular}




\begin{tabular}{|rrrr|rrrr|}
\hline $\mathrm{H}$ & -2.218298 & -0.312141 & -0.510522 & $\mathrm{H}$ & -1.774460 & -0.373048 & -1.169941 \\
$\mathrm{H}$ & -2.436970 & 0.372446 & 0.820910 & $\mathrm{H}$ & -0.991113 & -0.538959 & -2.500425 \\
$\mathrm{O}$ & 1.050865 & 0.680463 & 1.705752 & $\mathrm{H}$ & 2.001875 & -1.025838 & -0.178404 \\
$\mathrm{H}$ & 0.349372 & 1.235930 & 1.313984 & $\mathrm{H}$ & 3.196345 & -0.310896 & 0.666261 \\
$\mathrm{H}$ & 0.652767 & -0.209208 & 1.727970 & $\mathrm{H}$ & -0.731283 & 0.790128 & 1.113979 \\
$\mathrm{O}$ & -1.019723 & 2.085166 & 0.282840 & $\mathrm{H}$ & 0.647782 & 1.862492 & -0.168352 \\
$\mathrm{H}$ & -0.956628 & 1.685826 & -0.611274 & $\mathrm{H}$ & 0.018139 & -1.341005 & 1.560719 \\
$\mathrm{H}$ & -0.925325 & 3.036277 & 0.147740 & $\mathrm{H}$ & -0.570640 & -0.783060 & 2.874912 \\
\hline
\end{tabular}

\begin{tabular}{|rrrr|rrrr|}
\hline \multicolumn{7}{c}{ Neutral } & \multicolumn{3}{c|}{ Heptamer g2 } \\
\hline $\mathrm{O}$ & 2.835532 & 0.483332 & -0.350677 & $\mathrm{O}$ & -2.562613 & 0.608943 & -0.085812 \\
$\mathrm{H}$ & 3.742790 & 0.333431 & -0.059355 & $\mathrm{O}$ & -0.567522 & 0.854878 & -1.629399 \\
$\mathrm{H}$ & 2.310323 & 0.611730 & 0.476005 & $\mathrm{O}$ & 0.409061 & -1.528920 & -1.344509 \\
$\mathrm{O}$ & -0.261114 & -1.847975 & 1.196940 & $\mathrm{O}$ & 2.474413 & -0.679110 & 0.092850 \\
$\mathrm{H}$ & -0.431021 & -2.634655 & 1.729168 & $\mathrm{O}$ & 1.175413 & 1.889057 & -0.042735 \\
$\mathrm{H}$ & -1.149932 & -1.465416 & 0.990761 & $\mathrm{O}$ & 0.272152 & 0.527122 & 2.131381 \\
$\mathrm{O}$ & -1.063490 & 0.353274 & -1.875077 & $\mathrm{O}$ & -1.204827 & -1.559187 & 1.015958 \\
$\mathrm{H}$ & -1.226980 & 0.428034 & -2.822894 & $\mathrm{H}$ & -3.502504 & 0.695627 & -0.295777 \\
$\mathrm{H}$ & -0.272308 & -0.253773 & -1.779386 & $\mathrm{H}$ & -2.439648 & -0.248631 & 0.366157 \\
$\mathrm{O}$ & 0.951799 & -1.277507 & -1.356744 & $\mathrm{H}$ & 1.376257 & 2.833850 & 0.018638 \\
$\mathrm{H}$ & 1.732467 & -0.743057 & -1.091541 & $\mathrm{H}$ & 2.566133 & 0.277605 & -0.047081 \\
$\mathrm{H}$ & 0.657894 & -1.688325 & -0.522536 & $\mathrm{H}$ & 0.570190 & -2.141709 & -2.076683 \\
$\mathrm{O}$ & -2.487332 & -0.503165 & 0.410985 & $\mathrm{H}$ & -0.209589 & -0.089425 & -1.674129 \\
$\mathrm{H}$ & -2.218884 & -0.310807 & -0.509401 & $\mathrm{H}$ & -1.443297 & 0.809213 & -1.097115 \\
$\mathrm{H}$ & -2.437615 & 0.374252 & 0.821861 & $\mathrm{H}$ & 0.102073 & 1.381139 & -1.073126 \\
$\mathrm{O}$ & 1.052319 & 0.680838 & 1.705311 & $\mathrm{H}$ & 1.291313 & -1.360683 & -0.917213 \\
$\mathrm{H}$ & 0.351223 & 1.236455 & 1.313035 & $\mathrm{H}$ & 3.375923 & -1.019073 & 0.177444 \\
$\mathrm{H}$ & 0.653559 & -0.208518 & 1.728173 & $\mathrm{H}$ & -0.229466 & -0.274669 & 1.843269 \\
$\mathrm{O}$ & -1.017325 & 2.085905 & 0.281435 & $\mathrm{H}$ & 0.856189 & 1.594155 & 0.843252 \\
$\mathrm{H}$ & -0.955455 & 1.685514 & -0.612285 & $\mathrm{H}$ & -0.728604 & -1.915131 & 0.241396 \\
$\mathrm{H}$ & -0.920932 & 3.036648 & 0.145205 & $\mathrm{H}$ & -1.522711 & -2.332244 & 1.505020 \\
\hline
\end{tabular}

\begin{tabular}{|rrrr|rrrr|}
\hline \multicolumn{7}{c}{ Neutral } & \multicolumn{7}{c|}{ Heptamer g3 } \\
\hline $\mathrm{O}$ & 0.215569 & 2.588692 & 0.061223 & $\mathrm{O}$ & -0.220839 & 2.452805 & -0.053000 \\
$\mathrm{H}$ & -0.196685 & 3.317501 & -0.417705 & $\mathrm{O}$ & 2.797339 & -0.289353 & -0.534614 \\
$\mathrm{H}$ & 0.559803 & 1.981129 & -0.636208 & $\mathrm{O}$ & 1.263523 & 0.909067 & 1.281305 \\
$\mathrm{O}$ & -0.583495 & -1.688357 & -1.139265 & $\mathrm{O}$ & -1.260958 & 0.431674 & -1.720367 \\
$\mathrm{H}$ & -0.412390 & -2.524310 & -1.590308 & $\mathrm{O}$ & 0.507376 & -1.736754 & -1.049077 \\
$\mathrm{H}$ & -0.207191 & -1.790198 & -0.235074 & $\mathrm{O}$ & -0.234964 & -1.187562 & 1.534798 \\
$\mathrm{O}$ & -1.150719 & 0.708623 & 1.527871 & $\mathrm{O}$ & -2.582141 & -0.541934 & 0.582776 \\
$\mathrm{H}$ & -1.896793 & 0.487311 & 0.936943 & $\mathrm{H}$ & -0.606838 & 1.985265 & -0.820604 \\
$\mathrm{H}$ & -0.718310 & 1.474135 & 1.083682 & $\mathrm{H}$ & -0.094370 & 3.380059 & -0.294939 \\
$\mathrm{O}$ & -2.898861 & -0.208494 & -0.503688 & $\mathrm{H}$ & 0.413116 & -2.588722 & -1.502954 \\
$\mathrm{H}$ & -2.247146 & -0.843821 & -0.858894 & $\mathrm{H}$ & 0.191815 & -1.887744 & -0.134574 \\
$\mathrm{H}$ & -3.734505 & -0.689450 & -0.471746 & $\mathrm{H}$ & -3.418524 & -0.221344 & 0.945804 \\
$\mathrm{O}$ & 0.414928 & -1.381152 & 1.399761 & $\mathrm{H}$ & -2.437814 & -0.069072 & -0.256853 \\
$\mathrm{H}$ & -0.160424 & -0.560170 & 1.525761 & $\mathrm{H}$ & -0.677333 & -0.353016 & -1.742924 \\
$\mathrm{H}$ & 0.290541 & -1.900948 & 2.203706 & $\mathrm{H}$ & -1.641303 & 0.497306 & -2.608043 \\
$\mathrm{O}$ & 1.179334 & 0.666607 & -1.639704 & $\mathrm{H}$ & -1.191579 & -0.970288 & 1.350577 \\
$\mathrm{H}$ & 1.912154 & 0.293992 & -1.110549 & $\mathrm{H}$ & -0.231135 & -1.719524 & 2.345078 \\
$\mathrm{H}$ & 0.545156 & -0.069264 & -1.679081 & $\mathrm{H}$ & 1.933398 & 0.537818 & 0.631545 \\
$\mathrm{O}$ & 2.859953 & -0.504382 & 0.301314 & $\mathrm{H}$ & 2.131815 & -0.943316 & -0.871590 \\
$\mathrm{H}$ & 2.125844 & -0.886594 & 0.822774 & $\mathrm{H}$ & 0.650366 & 0.134006 & 1.513708 \\
$\mathrm{H}$ & 3.557350 & -1.170440 & 0.307483 & $\mathrm{H}$ & 0.703836 & 1.616395 & 0.782056 \\
\hline
\end{tabular}




\begin{tabular}{|rrrr|rrrr|}
\hline \multicolumn{3}{c}{ Neutral } & \multicolumn{3}{c|}{ Cation } \\
\hline $\mathrm{O}$ & 1.469463 & -1.469463 & 1.354420 & $\mathrm{O}$ & -1.953683 & 1.718129 & 1.007962 \\
$\mathrm{O}$ & -1.469463 & -1.469463 & -1.354420 & $\mathrm{O}$ & -1.980512 & 0.225513 & -1.063274 \\
$\mathrm{O}$ & 1.469463 & 1.469463 & -1.354420 & $\mathrm{O}$ & -1.736753 & -2.073800 & 0.009051 \\
$\mathrm{O}$ & -1.469463 & 1.469463 & 1.354420 & $\mathrm{O}$ & 0.354017 & 0.606468 & -2.079600 \\
$\mathrm{H}$ & 1.529572 & -1.529572 & 0.362414 & $\mathrm{O}$ & 1.632900 & 1.945010 & -0.203327 \\
$\mathrm{H}$ & -1.529572 & -1.529572 & -0.362414 & $\mathrm{O}$ & 2.298294 & -0.285132 & 1.359166 \\
$\mathrm{H}$ & 1.529572 & 1.529572 & -0.362414 & $\mathrm{O}$ & 1.622032 & -1.687667 & -0.824355 \\
$\mathrm{H}$ & -1.529572 & 1.529572 & 0.362414 & $\mathrm{O}$ & -0.355645 & -0.461153 & 1.864624 \\
$\mathrm{H}$ & 2.106304 & -2.106304 & 1.700599 & $\mathrm{H}$ & -2.059402 & 0.861817 & -0.272453 \\
$\mathrm{H}$ & -2.106304 & -2.106304 & -1.700599 & $\mathrm{H}$ & 0.893733 & 1.218162 & -1.507015 \\
$\mathrm{H}$ & 2.106304 & 2.106304 & -1.700599 & $\mathrm{H}$ & -1.283750 & -1.938646 & 0.859750 \\
$\mathrm{H}$ & -2.106304 & 2.106304 & 1.700599 & $\mathrm{H}$ & 2.316067 & -0.908155 & 0.593084 \\
$\mathrm{O}$ & 1.373308 & -1.373308 & -1.320801 & $\mathrm{H}$ & -2.690034 & 2.217929 & 1.386997 \\
$\mathrm{O}$ & -1.373308 & -1.373308 & 1.320801 & $\mathrm{H}$ & 0.449997 & 0.920720 & -2.990320 \\
$\mathrm{O}$ & 1.373308 & 1.373308 & 1.320801 & $\mathrm{H}$ & -2.439874 & -2.721186 & 0.156624 \\
$\mathrm{O}$ & -1.373308 & 1.373308 & -1.320801 & $\mathrm{H}$ & 3.098516 & -0.460724 & 1.874421 \\
$\mathrm{H}$ & 0.427057 & -1.515758 & -1.517803 & $\mathrm{H}$ & -1.092608 & 0.403662 & -1.524867 \\
$\mathrm{H}$ & -0.427057 & -1.515758 & 1.517803 & $\mathrm{H}$ & 2.170903 & 2.746416 & -0.251680 \\
$\mathrm{H}$ & 0.427057 & 1.515758 & 1.517803 & $\mathrm{H}$ & 0.640135 & -0.438964 & 1.904933 \\
$\mathrm{H}$ & 1.515758 & -0.427057 & -1.517803 & $\mathrm{H}$ & -1.962140 & -0.721250 & -0.684168 \\
$\mathrm{H}$ & -1.515758 & 0.427057 & -1.517803 & $\mathrm{H}$ & 1.301996 & -1.095789 & -1.527823 \\
$\mathrm{H}$ & 1.515758 & 0.427057 & 1.517803 & $\mathrm{H}$ & -1.525897 & 1.233791 & 1.733829 \\
$\mathrm{H}$ & -1.515758 & -0.427057 & 1.517803 & $\mathrm{H}$ & 2.091459 & 1.341615 & 0.415126 \\
$\mathrm{H}$ & -0.427057 & 1.515758 & -1.517803 & $\mathrm{H}$ & 1.985072 & -2.458929 & -1.281293 \\
\hline
\end{tabular}

\begin{tabular}{|rrrr|rrrr|}
\hline \multicolumn{7}{|c|}{ Octamer cube (s4) } \\
\hline $\mathrm{O}$ & 1.995080 & -0.177583 & -1.476324 & $\mathrm{O}$ & -0.843284 & -2.325108 & -0.511431 \\
$\mathrm{H}$ & 1.299705 & -0.888112 & -1.533790 & $\mathrm{O}$ & -2.129253 & -0.226946 & -0.849110 \\
$\mathrm{H}$ & 2.684698 & -0.427289 & -2.103326 & $\mathrm{O}$ & -2.332002 & 0.843278 & 1.479480 \\
$\mathrm{O}$ & -0.177583 & -1.995080 & 1.476324 & $\mathrm{O}$ & -0.280070 & 1.400324 & -1.768001 \\
$\mathrm{H}$ & -0.427289 & -2.684698 & 2.103326 & $\mathrm{O}$ & 1.736997 & -0.268387 & -1.668220 \\
$\mathrm{H}$ & -0.888112 & -1.299705 & 1.533790 & $\mathrm{O}$ & 0.635538 & -1.462830 & 1.554541 \\
$\mathrm{O}$ & -1.900895 & 0.046475 & 1.365195 & $\mathrm{O}$ & 0.354065 & 1.820422 & 0.906016 \\
$\mathrm{H}$ & -2.138416 & 0.114945 & 0.419791 & $\mathrm{O}$ & 2.767199 & 0.279844 & 0.868373 \\
$\mathrm{H}$ & -1.336447 & 0.828896 & 1.518972 & $\mathrm{H}$ & -0.238971 & -2.186954 & 0.260105 \\
$\mathrm{O}$ & -0.046475 & -1.900895 & -1.365195 & $\mathrm{H}$ & -1.258717 & -3.191273 & -0.406363 \\
$\mathrm{H}$ & -0.114945 & -2.138416 & -0.419791 & $\mathrm{H}$ & 3.662876 & 0.396775 & 1.214562 \\
$\mathrm{H}$ & -0.828896 & -1.336447 & -1.518972 & $\mathrm{H}$ & 2.278779 & 1.085285 & 1.110261 \\
$\mathrm{O}$ & 0.046475 & 1.900895 & -1.365195 & $\mathrm{H}$ & -1.544413 & 1.373829 & 1.685711 \\
$\mathrm{H}$ & 0.828896 & 1.336447 & -1.518972 & $\mathrm{H}$ & 0.163916 & 1.968087 & -0.053491 \\
$\mathrm{H}$ & 0.114945 & 2.138416 & -0.419791 & $\mathrm{H}$ & 1.510167 & -1.055208 & 1.410673 \\
$\mathrm{O}$ & 0.177583 & 1.995080 & 1.476324 & $\mathrm{H}$ & 0.713552 & -1.944565 & 2.389020 \\
$\mathrm{H}$ & 0.888112 & 1.299705 & 1.533790 & $\mathrm{H}$ & -1.649311 & -1.149172 & -0.700399 \\
$\mathrm{H}$ & 0.427289 & 2.684698 & 2.103326 & $\mathrm{H}$ & -1.494488 & 0.382908 & -1.327896 \\
$\mathrm{O}$ & -1.995080 & 0.177583 & -1.476324 & $\mathrm{H}$ & 0.518968 & 0.831501 & -1.943828 \\
$\mathrm{H}$ & -1.299705 & 0.888112 & -1.533790 & $\mathrm{H}$ & -0.400902 & 1.961181 & -2.547910 \\
$\mathrm{H}$ & -2.684698 & 0.427289 & -2.103326 & $\mathrm{H}$ & -2.310216 & 0.193707 & 0.054954 \\
$\mathrm{O}$ & 1.900895 & -0.046475 & 1.365195 & $\mathrm{H}$ & -3.085287 & 1.267607 & 1.911049 \\
$\mathrm{H}$ & 2.138416 & -0.114945 & 0.419791 & $\mathrm{H}$ & 2.234800 & -0.769258 & -2.326991 \\
$\mathrm{H}$ & 1.336447 & -0.828896 & 1.518972 & $\mathrm{H}$ & 2.340445 & -0.126161 & -0.914322 \\
\hline
\end{tabular}

\begin{tabular}{|lrrr|}
\hline \multicolumn{3}{|c|}{$\left.\mathrm{H}_{2} \mathrm{O}\right)_{24}$} \\
\hline \multicolumn{3}{|c|}{ Neutral } \\
\hline $\mathrm{O}$ & -1.730527 & 0.000000 & 2.666972 \\
$\mathrm{H}$ & -1.387569 & 0.000000 & 1.773536 \\
$\mathrm{H}$ & -2.681413 & 0.000000 & 2.558969 \\
$\mathrm{O}$ & -1.730527 & 0.000000 & -2.666972 \\
$\mathrm{H}$ & -1.387569 & 0.000000 & -1.773536 \\
\hline
\end{tabular}




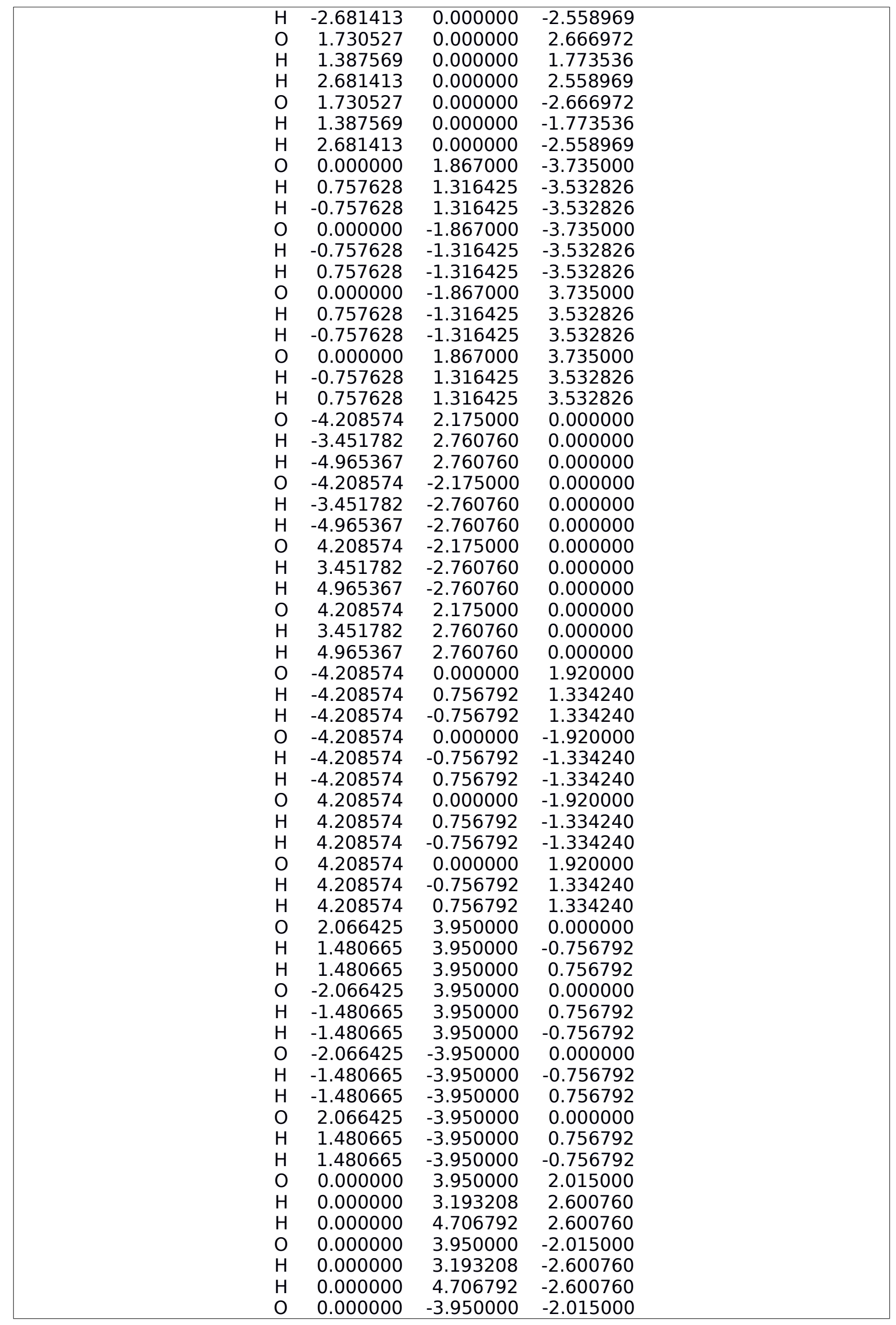




\begin{tabular}{llll|}
$\mathrm{H}$ & 0.000000 & -3.193208 & -2.600760 \\
$\mathrm{H}$ & 0.000000 & -4.706792 & -2.600760 \\
$\mathrm{O}$ & 0.000000 & -3.950000 & 2.015000 \\
$\mathrm{H}$ & 0.000000 & -3.193208 & 2.600760 \\
$\mathrm{H}$ & 0.000000 & -4.706792 & 2.600760 \\
\hline
\end{tabular}

